\title{
Localization of type IV collagen alpha chains in tooth germ development
}

\author{
Naoki Katase ${ }^{1)}$, Hitoshi Nagatsuka ${ }^{1)}$, Keisuke Nakano ${ }^{1)}$,Ryo Tamamura ${ }^{1)}, X i e$ Wel-li²), \\ Yukiteru Matsuoka ${ }^{3)}$, and Noriyuki Nagai ${ }^{1)}$ \\ 1) Department of Oral pathology and Medicine, Graduate School of Medicine, Dentistry and Pharmaceutical Sciences, Okayama \\ University, Okayama, Japan \\ 2) Faculty of Dentistry, Harbin Medical University, Harbin, China \\ 3) Japan Institute for Advanced Dentistry, Osaka, Japan
}

\begin{abstract}
The morphogenesis and morphological development of molar are regulated by the interaction between odontogenic epithelium and mesenchymal tissues. And Type IV collagen molecules, the major component of the dental basement membrane, are composed of three alpha chains. Using indirect immunofluorescence on cryosections, we characterized the changes in protein localization of alpha 1 to 6 chains of type IV collagen during molar development in mice molar and human tooth germ.

ICR mouse embryos (detection of vaginal plug: day 0) and newborn mice were used. Human tooth germ was kindly provided by Dr.Yamamoto of Ohu University. Rat monoclonal antibodies recognizing type IV collagen alpha chains were raised against synthetic peptides of non-consensus amino acid sequences of the human and mice alpha (IV) chains. These monoclonal antibodies were kindly provided by Shigei medical research institute. Both mice and human tooth germ, we detected conversions in localization and composition of type IV collagen alpha chains working with tooth germ development. These results imply that there are time and site specificity, which may be involved in tooth morphogenesis and the tooth germ development, in type IV collagen alpha chains.
\end{abstract}

\section{Introduction}

The morphogenesis and morphological development of teeth are regulated by epithelium-mesenchymal interactions, and the basement membrane plays a role in mediating these interactions.

Type IV collagen molecules, the major component of the dental basement membrane, is composed of three alpha chains. Using indirect immunofluorescence on cryosections, we characterized the changes in protein localization of alpha 1 to alpha 6 chains of type IV collagen during molar development in mice molar and human tooth germ.

\section{Preparation of Tissues}

\section{Material and Method}

ICR mouse embryos (detection of vaginal plug: day 0) and newborn mice were used. The heads of mice at embryonic day 11 to 17 and postnatal day 1 were embedded and frozen in liquid nitrogen, then cut into 4 ìm sagittal sections.

One set of the cryosections were stained by hematoxylin and eosin for observation.

Human tooth germ was kindly provided by Dr.Yamamoto of Ohu University.

2. Monoclonal antibodies Rat monoclonal antibodies recognizing type IV collagen alpha chains were raised against synthetic peptides of non-consensus amino acid sequences of the human alpha(IV) chains and mice alpha(IV)chains. Their specificity against the individual human alpha (IV) chains was confirmed by ELISA and immunoblotting. These monoclonal antibodies were kindly provided by Shigei medical research institute.

\section{Results}

1. Mouse tooth germ

Samples collected on embryonic days (ED) 11, 13, 15 and postnatal day (PD) 1 corresponded to the dental lamina, bud stage, cap stage, and bell stage.

(1)Dental lamina (ED11) and bud stage (ED13)

Antibodies against alpha1, 2, 4, 5 and 6 chains stained the basement membrane along the dental lamina (ED11) but antibody against alpha3 chain was negative during all odontogenic epithelial development.

(2)Cap stage (ED15) and early bell stage The inner enamel epithelium and enamel knot of the enamel organ were positive for alpha1, 2 and 4 chains, and the outer enamel epithelium were positive for alpha1,2 but not alpha4 chain. The dental lamina of molar germ was positive for alpha1, 2, 5 and 6.

3)Late Bell Stage with Initial Matrix Formation (PD1)In the late bell stage, immunostaining with antibodies against alpha1,2, and 4 chains remained continuous along the BM of the inner enamel epithelium, while only alpha1 and 2 chains were demonstrated in the outer enamel epithelium. When functional ameloblasts started

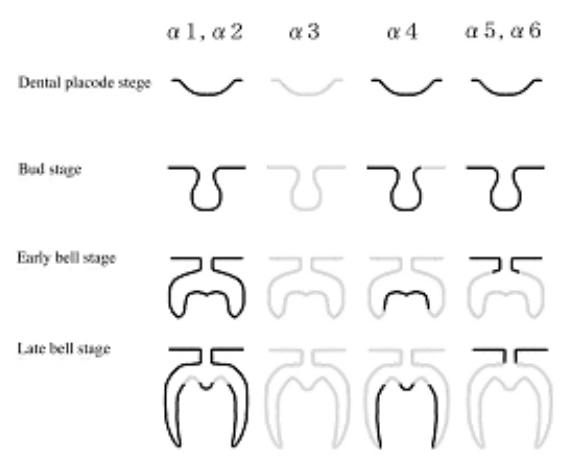

Fig.1. Summary of mouse tooth germ 


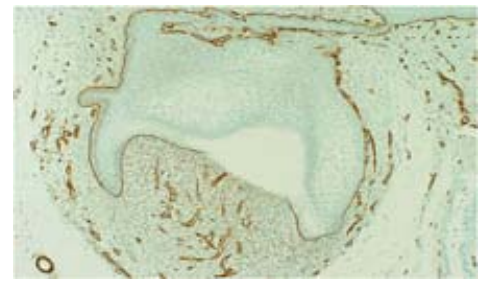

Fig.2. Human tooth germ $(\alpha 1)$

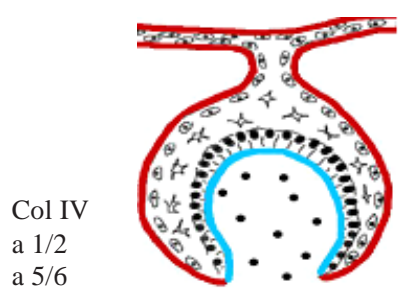

Fig.3. Summary of human tooth germ to secrete the initial enamel matrix in the cuspal area, the distribution of alpha1, 2 and 4 chains in inner enamel epithelium became discontinuous and disappeared towards the tip of the cusp. At this stage, the oral epithelium was positive for alpha1, 2, 5 and 6 chains. The changes in distribution of alpha1 to alpha 6 chains of type IV collagen in each stage and area of mouse molar germ morphogenesis are summarized in Fig.1.

2. Human tooth germ

Bell stage human tooth germ was used.

Odontogenic epithelium basement membrane was positive for alpha 1, 2, 5 and 6 chains. Alpha 4 was positive only in inner enamel epithelium. And alpha 1, 2, 5 and 6 chains were positive in outer enamel epithelium. In inner enamel epithelium, all alpha chains other than alpha 3 were positive. In the vessels and oral mucosa epithelium, alpha 1 and 2 were most positive. (Fig.2) These results are summarized in Fig.3.

\section{Discussion and Conclusion}

These results suggest that type IVcollagen alpha chains have stage- and position- specific distributions.

As to mouse tooth germ, In the dental lamina and bud stage, the oral epithelium expressed alpha 1, 2, 5 and 6 chains. And the dental lamina expressed alpha 4 chain in additional to the above four chains. In the cap and early bell stage, the inner enamel epithelium of molar germ expressed alpha 1,2 and 4 chains, while the outer enamel epithelium expressed only alpha 1 and 2 chains, resembling that of the capillary. The distribution of alpha 1, 2 and 4 chains in inner enamel epithelium gradually disappeared with the progression of dentinogenesis. The dental lamina of molar epithelium expresses alpha 1,2, 5 and 6 from the cap stage to bell stage, resembling fetal oral mucosa. As to human tooth germ, in the bell stage,

The basement membrane of tooth germ epithelium expressed alpha 1, 2, 5 and 6 chains. And inner enamel epithelium expressed alpha 3 chain in additional to above four chains. Outer enamel epithelium expressed alpha 1, 2, 5 and 6 chains. This stage- and site- specific distributions of alpha subunits of type IV collagen in molar development may be related to molar morphogenesis and cytodifferentiation.

\section{References}

1. Nakano K. Localization of type IVcollagen $\alpha 1-6$ chains in basement membrane during mouse development. 岡山歯誌 18,69-82,1999

2. Fujii $\mathrm{H}$ et al.: Differential expression of type IV collagen alpha 1 to alpha 6 chains in basement membrane of human tooth germ and odontogenic tumors. J.Hard Tissue Biology.13 (2):103-109, 2004 